\title{
Editorial
}

\section{Gene therapy for Parkinson's disease: current knowledge and future perspective}

Parkinson's disease continues to be the only neurodegenerative disorder to be treated successfully using transmitter replacement strategy. However, experience over the past three decades has clearly shown that classical pharmacotherapy is suboptimal since motor response fluctuations and dyskinesias complicate the course of most patients with advanced stages of the disease. ${ }^{1}$ Furthermore, this approach provides only symptomatic relief; no current therapy has been definitively shown to slow down the relentless loss of nigral dopaminergic neurons. Thus, an urgent need exists to develop novel treatment strategies that would target the basic pathogenetic defect in Parkinson's disease in an effort to arrest or retard the degenerative process. Although fetal nigral tissue implantation appears promising, it is associated with a number of logistical and ethical issues that tend to limit the availability of this procedure. More practical and reproducible means for delivering therapeutic genes would allow larger scale systematic evaluation of efficacy.

Gene therapy holds considerable potential for the treatment of central nervous system (CNS) disorders in general. Parkinson's disease is a particularly appropriate target for gene therapy since the pathology is fully characterized and well circumscribed, animal models are available and several candidate genes, known to favorably impact dopaminergic neurons in vitro and in vivo, are already cloned. In addition, the search for genes responsible for familial forms of Parkinson's disease is well under way with a locus on chromosome 4q21-q23 already identified. ${ }^{2}$

While little doubt remains about the timeliness of genebased therapy for Parkinson's disease, the development of gene delivery methods to achieve this objective is still in its formative stages. Mainly due to delivery issues, research on gene therapy for CNS disorders has generally lagged behind that for systemic disorders. The inaccessibility of the brain as well as its structural and physiological complexities have been formidable obstacles. To date, clinical gene therapy has been attempted in only two CNS disorders, namely brain tumors and the rare Canavan disease.

The current state of research in gene therapy for Parkinson's disease is typical of early development efforts for any novel therapeutic modality. Uncertainties about optimal gene transfer methodology have prompted testing all known strategies by different research groups. Direct introduction of a therapeutic gene into the brain using the in vivo approach has employed a variety of methods including the injection of naked DNA, liposome-associated DNA or various viral vectors expressing a therapeutic gene. For the latter, different viral vector systems are being used in the experimental animal including those derived from herpes simplex virus, adenovirus and adeno-associated virus. Although retroviruses generally require replicating cells for their life cycle, a lentiviral vector based on the human immunodeficiency virus capable of infecting striatal and hippocampal neurons and expressing the transgene in adult rats has recently been reported. ${ }^{3}$ In general, these viral vectors are designed to be devoid of their cytopathogenic genes which are replaced by the therapeutic gene(s). Despite a large body of investigation focusing on in vivo gene therapy for the brain, several issues remain of concern including cellspecific transgene expression, duration of expression, recombination with latent wild-type virus resulting in virulent viral strains and cytotoxicity, induction of an immune response by the host and insertional mutagenesis. Alternatively, ex vivo engineering of appropriate cells followed by implantation at a specific brain region is being tested using a variety of cell types. These include autologous skin fibroblasts, myoblasts, Schwann cells and polymer-encapsulated cell lines. The latter allows the implantation of engineered xenogeneic cells shielded from immune attack while being able to secrete the transgene product out of the polymer device. ${ }^{4}$ In addition, the physical barrier of the device prevents uncontrolled cellular proliferation, the device can be explanted when desired and suicide genes can be incorporated in the cells to allow their elimination if needed. The third and less well explored strategy for Parkinson's disease is gene modulation therapy, whereby the expression level of an endogenous gene within the brain is regulated by various methods for therapeutic intent, for example to augment the expression of tyrosine hydroxylase (TH) or neurotrophic factors.

The ideal in vivo gene transfer method should produce long-term, cell-type specific gene expression at adequate levels but not result in cytotoxicity, insertional mutagenesis, immune reaction to viral antigens or the emergence of virulent viral particles. Unfortunately, none of the vectors used today in their present form satisfy all these criteria. Considerable improvements are still required to make these vectors safe and effective. And the ideal requirements for ex vivo gene transfer include expression of the transgene for a long time and at adequate levels without forming tumors or inducing an immune reaction while ultimately integrating with the host brain circuitry. 
Several advantages and disadvantages are unique to the ex vivo and in vivo gene transfer methods. In addition to allowing characterization of the engineered cells before implantation, the ex vivo approach provides the possibility of either retrieving the graft especially with encapsulated cell implants or killing the cells in situ by inducing an incorporated suicide gene when the biological effects are no longer wanted. However, there is generally more disturbance with the normal cytoarchitectural circuitry of the brain with implantation except perhaps when progenitor neural cells are used. ${ }^{5}$ Gene product delivery is limited by the process of diffusion through the brain parenchyma with the ex vivo approach and by both transfection efficiency and tissue diffusion with the in vivo approach. While the generation of an immune response is not an issue with the implantation of autologous or encapsulated cells, it could be a significant problem with direct introduction of viral vectors into brain cells particularly with adenoviral vectors. Furthermore, the risk of helper virus is a frequent safety concern with the in vivo approach. Finally, the expression of functional intracellular survival genes, such as those that modulate apoptotic cascades, are presently achievable only with the direct introduction of transgenes into brain cells using the in vivo method.

The development of gene-based therapies for Parkinson's disease requires two primary considerations: what gene should be targeted and how to deliver it. The development of a rational strategy for gene-based therapy for any disorder in general and for Parkinson's disease in particular clearly requires first the identification of the defective gene and characterization of the normal gene product. Elucidation of the functional properties of a protein is essential before a feasible plan can be formulated. For example, if the mutated gene is a developmental one, its delivery into the mature brain would not be expected to be beneficial. Alternatively, if the defective gene is tightly controlled, then the requisite regulatory elements should be incorporated in the vectors. Finally, understanding the mechanism by which the mutation alters the phenotype is crucial, particularly knowing whether it results in loss of function or gain of function. In case of the latter scenario, adding a normal copy of the gene would not eliminate the defective dominant mutant phenotype. While the genes responsible for Parkinson's disease remain to be identified, current alternatives include either augmentation of dopaminergic transmission or attempting to preserve residual nigral dopamine neurons.

Delivery of the cDNA for $\mathrm{TH}$, the rate-limiting enzyme in dopamine biosynthesis, into the striatum confers the ability to synthesize and release dopamine which can interact with nearby dopaminergic receptors. Thus, this gene product provides relief of Parkinsonian symptoms similar to that occurring with oral levodopa treatment. In addition, the introduction of $\mathrm{TH}$ may exert an indirect neuroprotective effect, since loss of dopaminergic neurons in Parkinson's disease leads to a compensatory acceleration in dopamine synthesis and catabolism in residual nigrostriatal terminals, resulting in increased generation of potentially damaging free radicals. To the extent that the delivery of $\mathrm{TH}$ augments dopamine synthesis in other dopa decarboxylase-containing cells, increased dopamine turnover and thus oxidative stress in residual nigrostriatal neurons should diminish. Yet, perhaps the best argument for delivering the TH cDNA at this early stage of the field is its presumed ability to provide relatively early outcome measures that can be readily assessed behaviorally. The latter provides an easier means of evaluating the efficacy of a particular gene delivery method than studies employing gene products with neuroprotective or neurorestorative potential which require long observation periods and large sample sizes.

The two main gene-based strategies currently available to preserve dopaminergic neurons involve either neurotrophic factors or anti-apoptotic genes. Mounting evidence points to the neuroprotective efficacy of brainderived neurotrophic factor (BDNF) and glial cell linederived neurotrophic factor (GDNF) as well as the neurorestorative potential of GDNF in neurotoxin induced experimental Parkinsonism. In addition, recent findings support an apoptotic mechanism of neuronal death in the substantia nigra of Parkinsonian patients. ${ }^{6}$ Thus, the cDNA's coding for anti-apoptotic proteins, such as bcl-2 or $b c l-x_{L}$, are good candidates.

The next primary issue is delivery, specifically whether to use the in vivo or ex vivo approach. This decision depends partly on the properties of the particular gene product to be transferred. For example, genes encoding extracellular neurotrophic factors could be delivered by either approach while those encoding intracellular antiapoptotic proteins currently require in vivo methods. Secondary considerations include targeting the gene into an appropriate brain region and cell type, achieving sustained and adequate expression levels, using a regulatable promoter and incorporating a suicide gene. Finally, as applied to any therapeutic modality, assessment of the risk:benefit ratio and comparison to alternative existing therapies are essential. Generally, although conventional therapies for Parkinson's disease are effective initially, the quality of life of these patients deteriorates markedly in later stages which prompts many to undergo ablative neurosurgical procedures.

The availability of animal models for Parkinson's disease has facilitated the initiation of preclinical gene transfer studies for this disease earlier than for other neurodegenerative disorders. The 6-hydroxydopamine lesion of nigral neurons in rats resulting in contralateral rotational behavior upon administration of dopaminergic agents is well characterized. Although this model is relatively easy and inexpensive to generate and use, the behavioral outcome of the lesion is far from the human disease. The vulnerability of nigral dopamine neurons in mice and especially in nonhuman primates to the neurotoxin 1-methyl-4-phenyl-1,2,3,6-tetrahydropyridine (MPTP) provides a second approach, which in the case of primates much more closely approximates the clinical phenomenology of human Parkinsonism. The predictive value of both models has been repeatedly validated for dopaminergic agents: antiparkinsonian drugs that appropriately modify motor behavior in these models have characteristically proven symptomatically effective in patients. However, the validity of these models to predict the efficacy of neuroprotective or neurorestorative strategies has yet to be demonstrated. Indeed, lesioning in either model is generally done acutely, producing rapid severe injury to nigral dopamine neurons which is clearly different than the slow progressive nature of the degenerative process in Parkinson's disease. Conceivably, such animal models might have a higher 
predictability index when used for testing neurorestorative strategies at which point earlier steps in the pathogenetic cascade might not be as critically relevant as when applied to the evaluation of neuroprotective approaches. Furthermore, the design of 'proof-of-concept' experiments in animal models of disease in general should anticipate more heterogeneity of response in the human population.

Notwithstanding the important advances currently being made in gene therapy, the concept of modulating a genetic disorder using conventional pharmaceutical modalities is clearly more attractive from the clinical management perspective than injecting viral vectors or transplanting cells. The development of small molecules based on genetic information is possible and certainly easier, less costly and perhaps safer. A number of strategies can be formulated by which such small molecules could conceivably make an impact on a genetic defect. Ample examples of such therapies exist in medicine, particularly in endocrine and metabolic disorders. Small molecules could theoretically modulate an enzymatic pathway, act as a receptor ligand, modulate transcription of a gene or protein-protein interaction or target alternative pathways in the cell.

While major improvements must occur before gene therapy for Parkinson's disease becomes of clinical value, continued preclinical testing using several different approaches is prudent for two major reasons. First, it is not clear at present which approach or vector will prove optimal. Second, different strategies might be needed for different genes; and if Parkinson's disease proves to be due to multiple gene defects, as is the case with Alzheimer's disease, a unified approach for all patients and families would not be suitable.

MM Mouradian and TN Chase Experimental Therapeutics Branch National Institute of Neurological Disorders and Stroke National Institutes of Health

Bethesda, MD, USA

\section{References}

1 Mouradian MM, Chase TN. Improved dopaminergic therapy of Parkinson's disease. In: Marsden CD, Fahn S (eds). Movement Disorders 3. Butterworth Heinemann: Oxford, 1994, pp 181-199.

2 Polymeropoulos MH et al. Mapping of a gene for Parkinson's disease to chromosome 4q21-q23. Science 1996; 274: 1197-1199.

3 Naldini L et al. In vivo gene delivery and stable transduction of nondividing cells by a lentiviral vector. Science 1996; 272: 263267.

4 Winn SR et al. Behavioral recovery following intrastriatal implantation of microencapsulated PC12 cells. Exp Neurol 1991; 113: 322-329.

5 Gage FH et al. Survival and differentiation of adult neuronal progenitor cells transplanted to the adult brain. Proc Natl Acad Sci USA 1995; 92: 11879-11883.

6 Mochizuki H, Goto K, Mori H, Mizuno Y. Histochemical detection of apoptosis in Parkinson's disease. J Neurol Sci 1996; 137: $120-123$. 